\title{
Diagnostic utility of Sudoscan for detecting bortezomib-induced painful neuropathy: a study on 18 patients with multiple myeloma
}

\author{
Alessandro Allegra ${ }^{1}$, Vincenzo Rizzo ${ }^{2}$, Vanessa Innao ${ }^{1}$, Angela Alibrandi ${ }^{3}$, Anna Mazzeo², \\ Rossana Leanza ${ }^{1}$, Carmen Terranova ${ }^{2}$, Luca Gentile², Paolo Girlanda², Andrea Gaetano Allegra ${ }^{1}$, \\ Andrea Alonci ${ }^{1}$, Caterina Musolino ${ }^{1}$
}

\begin{abstract}
${ }^{1}$ Division of Hematology, Department of Human Pathology in Adulthood and Childhood "Gaetano Barresi", University of Messina, Messina, Italy

2Department of Clinical and Experimental Medicine, University of Messina, Messina, Italy

${ }^{3}$ Department of Economics, Unit of Statistical and Mathematical Sciences, University of Messina, Messina, Italy
\end{abstract}

Submitted: 28 July 2019; Accepted: 16 November 2019

Online publication: 11 January 2021

Arch Med Sci 2022; 18 (3): 696-703

DOI: https://doi.org/10.5114/aoms/114269

Copyright @ 2021 Termedia \& Banach

\section{Abstract}

Introduction: In the past few years, treatment of multiple myeloma has undergone a deep change for the employment of novel treatment comprising proteasome inhibitors. Bortezomib is a first-line drug in therapy of multiple myeloma. The onset of peripheral neuropathy is a dose-limiting collateral effect of the drug. This neuropathy is a distal symmetric neuropathy that affects both large and small fibers. Nerve conduction study (NCS) can be used for the diagnosis of bortezomib neuropathy, but this technique demonstrates alterations of the large nerve fibers. Sudoscan is a novel technique utilized to offer an evaluation of sudomotor function. The main objective of this study was to compare the sensitivity and diagnostic specificity of Sudoscan with respect to the nerve conduction study after bortezomib treatment.

Material and methods: A total of 18 multiple myeloma patients were studied, $10(55.5 \%)$ men and 8 (44.5\%) women. Patients were analyzed at baseline and after 6 months of treatment with bortezomib. Subjects were submitted to nerve conduction study and electrochemical skin conductance evaluation with the Sudoscan device. Patients were also submitted to a clinical measure of pain and neuropathy.

Results: At baseline NCS showed that only the mean sural SAP amplitude was below the 2SD lower limit of normal in $3(16.7 \%)$ patients, while at same time we found an alteration of Sudoscan profiles in $2(11.1 \%)$ patients. After 6 months of treatment, the NCS profiles were altered in $13(72.2 \%)$ patients, and the Sudoscan profiles were modified in $11(61.1 \%)$ subjects. Conclusions: Our results suggest that Sudoscan can be considered for the diagnosis of bortezomib-induced neuropathy. It is objective, reproducible, and surely easier than the traditional nerve conduction study. Sudoscan may be a useful help to manage the therapeutic interventions in multiple myeloma.

Key words: multiple myeloma, bortezomib, peripheral neuropathy, Sudoscan, nerve conduction study, sudomotor function.

\author{
Corresponding author: \\ Prof. Alessandro Allegra \\ Division of Hematology \\ Department \\ of Human Pathology in \\ Adulthood and Childhood \\ "Gaetano Barresi" \\ University of Messina \\ Messina, Italy \\ E-mail: aallegra@unime.it
}




\section{Introduction}

In the past few years, treatment of multiple myeloma (MM) has undergone a deep change for the employment of novel treatment comprising proteasome inhibitors (PIs), immunomodulatory drugs, epigenetic therapy, and monoclonal antibodies [13]. This event, combined with the frequent employment of autologous stem cell transplant and the progress in the knowledge of MM biology [4], has led to improved overall survival for MM subjects [5].

Bortezomib is the first of a novel group of drugs known as PIs [6] and is a first-line chemotherapeutic in therapy of newly diagnosed MM (NDMM).

Bortezomib is a boronic acid dipeptide which reversibly blocks the $20 \mathrm{~S}$ catalytic subunit of the $26 \mathrm{~S}$ proteasome. Proteasome inhibition has been demonstrated to block crucial cell pathways, comprising the ubiquitin-proteasome pathway and nuclear factor $\kappa \mathrm{B}(\mathrm{NF}-\kappa \mathrm{B})$, JNK and $\mathrm{p} 53$ function, to cause cell death. Multiple myeloma cells are more susceptible to the action of the drug, undergoing cell death more promptly than normal cells. Moreover, bortezomib can induce G2M phase cell cycle arrest and tubulin polymerization, and axonal transport inhibition $[7,8]$.

The use of bortezomib treatment was associated with the onset of peripheral neuropathy as a frequent, dose-limiting collateral effect [9]. Several studies describe elevated occurrence of peripheral neuropathy (more than 70\%) after bortezomib treatment [10]. Moreover, numerous studies have demonstrated that although up to $75 \%$ of subjects with bortezomib-induced painful neuropathy (BIPN) had resolution of symptoms within 2-3 months of treatment termination or dose modification [11], 25\% of BIPN subjects continue to have symptoms also after dose reduction or drug interruption (13-20 months) [12].

BIPN is generally a distal symmetric neuropathy which affects both large and small fibers although in many cases symptoms of small-fiber damage predominate. BIPN is described as a condition characterized by several symptoms such as hypoesthesia and heat hypersensitivity with poor motor involvement [13]. Its main characteristic is presence of grave neuropathic pain which is often devastating. BIPN is not treatable with any treatment and critical forms require bortezomib suspension $[14,15]$.

Nerve conduction study (NCS) can be used to make a diagnosis of BIPN $[16,17]$, but generally this technique can demonstrate alterations of the large nerve fibers and not those implicating the small fibers.

Modifications in the peripheral autonomic nervous system are an initial expression of distal small fiber neuropathy [18]. Sudomotor alteration is one of the earliest anomalies in small fiber dis- orders. Sweat glands are innervated by unmyelinated cholinergic sympathetic C-fibers and several reports have demonstrated a decrease in the epidermal C-nerve fibers in diabetic subjects with small fiber neuropathy $[19,20]$. Hence, evaluation of sudomotor function may be an efficacious method to study peripheral small fiber neuropathy in different settings of patients [21].

Sudoscan is a new and modern evolution of electrophysiological methods aiming to provide a non- invasive and quantitative evaluation of the sudomotor function $[22,23]$. It measures in microsiemens $(\mu \mathrm{S})$ the electrochemical skin conductance derived from the reaction between the sweat chloride and the nickel electrodes. Recently, Sudoscan has been considered a helpful tool in the assessment of small fiber neuropathy (SFN) caused by diabetes [22], mitochondria dysfunction [24], and amyloid deposition [25].

The main objective of this study was to evaluate sensitivity of the NCS and Sudoscan, for detecting early peripheral nerve involvement in $\mathrm{MM}$ patients after treatment with bortezomib. Moreover, this study aims to evaluate whether this measure could be used as a reliable tool to detect longitudinal changes in the small fiber function in these patients after bortezomib treatment. The results of our study would seem to confirm the possibility that Sudoscan may be a useful means of evaluating the BIPN.

\section{Material and methods}

This study was carried out in accordance with the principles of the Declaration of Helsinki and the Local Ethic Committee approved this study protocol before the initiation of any study-related procedures; informed consent was obtained from every participant.

This study was conducted from May 1, 2017 to January 20, 2019. A total of 18 NDMM patients were studied, 10 (55.5\%) men and 8 (44.5\%) women. The median age was 70.0 years (range: 39-87). International Staging System (ISS) I, II, and III disease stages at diagnosis were prevalent in $5(27.8 \%), 6(33.3 \%)$, and 7 (38.9\%) of the participants, respectively.

Exclusion criteria were amputation of legs or arms, symptoms limited to the upper extremity due to cervical radiculopathy or upper extremity entrapment neuropathy, implanted electrical devices, diabetes mellitus, and previous peripheric polyneuropathy (PPNP) history.

The paraprotein class was immunoglobulin G (lgG) in 13 (72.2\%) patients, and IgA in 5 (27.8\%) patients. Light chain was $\kappa$ in $10(55.6 \%)$ patients, and $\lambda$ in 8 (44.4\%) patients.

At baseline routine laboratory tests consisted of complete blood count, and blood chemistry 
including $\beta 2$ microglobulin (Beta2m), serum albumin, calcemia, and renal function. Physical examination and skeletal X-rays were also performed. Fourteen (77.8\%) subjects had lytic bone lesions, while only $4(22.2 \%)$ did not present them.

Five $(27.7 \%)$ patients received bortezomib, thalidomide and dexamethasone (VTD) chemotherapy, while 13 (72.2\%) were treated with the bortezomib, melphalan and prednisone (VMP) regimen.

At baseline and after 6 months of treatment patients were submitted to a clinical measure of pain and neuropathy. The three scales employed were the Douleur Neuropathique en 4 Questions (DN4), the Total Neuropathy Score (Clinical) (TNSC), and the Pain Intensity Numerical Rating Scale/PINRS.

The full version of the DN4 comprises 7 self-report items and 3 clinical examination questions. Scores range from 0 to 10 and a score of 4 or higher is considered positive for neuropathic pain [26].

The TNSc combines data acquired from quantitative sensory examinations, and scoring of signs, thus supplying a single measure to evaluate neuropathy [27].

For the PINRS the patients are requested to classify their pain with a numerical value indicating its intensity. The PINRS scale ranges from 0 to 10 , with 10 indicating the worst possible pain [28]

The electrochemical skin conductance (ESC) was evaluated in the MM subjects at baseline and after 6 months of treatment, with the Sudoscan device (Impeto Medical: Paris, France) [29].

All sudomotor function tests were performed in a quiet and temperature-controlled autonomic laboratory with ambient air humidity (room temperature: $22-23^{\circ} \mathrm{C}$, room humidity $\left.40-60 \%\right)$. Subjects were in an upright position during the Sudoscan testing. The electrochemical principle involves the induction of a chloride-based electrochemical current after activation of sweat glands by a low-voltage current $(<4 \mathrm{~V})$ [30]. Due to the isolator function of the corneal stratum of the epidermis, it is expected that the measured net current corresponds to the local sweat response. The ESC in microsiemens $(\mu \mathrm{S})$ that represents the chloride ion current is calculated by the ratio of extrapolated current and constant direct current. ESC was measured at both hands and feet by placing the palms and soles on stainless steel electrodes for $2 \mathrm{~min}$. The measurement was repeated twice, and the average ESC was calculated. Low ESC indicates a high risk of a somatosensory neuropathy [31]. Based on a previous study of Vinik and colleagues, an ESC of $>70 \mu \mathrm{S}$ (feet)/> $60 \mu \mathrm{S}$ (hands) is considered to indicate normal sudomotor function while an ESC of 50-70 $\mu \mathrm{S}$ (feet)/40-60 $\mu \mathrm{S}$ (hands) and of $<50 \mu \mathrm{S}$ (feet) $/<40 \mu \mathrm{S}$ (hands) is suggestive of moderate and severe sudomotor dysfunction respectively [29].
NCS consisted of sensory nerve conduction of the sural and ulnar nerve and motor nerve conduction of the peroneal, tibial and ulnar nerve. The voltage applied to subjects for the nerve conduction study was about 90-150 V.

NCS was conducted according to internationally accepted standards, and the 2SD lower limit of normal of local reference values was used for statistical testing and to determine the percentage of abnormal measurements [32].

\section{Power and sample size}

Sample size calculation is based on the primary outcome, which is the incidence of multiple myeloma. We assume for this study a known population incidence of $0.00875 \%$ (since in Italy the incidence is estimated to be 8.75 new cases per 100,000 inhabitants) and expected incidence of $9 \%$ (this is the anticipated incidence of $M M$ for our study group). In order to guarantee a power of power of $80 \%$, and a two-sided significance level of $5 \%$, the sample size calculation suggests that the minimum number of subjects is 8 . In our study we enrolled 18 patients.

\section{Statistical analysis}

Numerical data are expressed as mean and standard deviation (S.D.) and categorical variables as number and percentage. The non-parametric approach was used since the numerical variables were not normally distributed, as verified by the Kolmogorov-Smirnov test. The Wilcoxon test was applied to perform comparisons between times of observation (baseline vs. 6 months) with reference to numerical parameters (DN4, PI-NRS and TNSc); the McNemar test was applied for dichotomous data (NCS and Sudoscan). The Spearman correlation test was applied to assess the existence of any significant interdependence between analyzed parameters. Finally, the Pearson $\chi^{2}$ test was used to analyze differences between the two techniques, NCS and Sudoscan, with reference to the percentages of alteration, both at baseline and after 6 months of treatment.

Statistical analyses were performed using IBM SPSS for Windows, Version 22 (Armonk, NY, IBM Corp.). A $p$-value smaller than 0.05 was considered statistically significant.

\section{Results}

After treatment the overall response rate to treatment was $83.3 \%$. Complete responses were observed in 5 of 18 patients (27.7\%), very good partial responses in 10 (55.5\%) subjects, and 3 (16\%) patients experienced progression of disease.

At baseline the NCS profiles showed that only the mean sural nerve sensory nerve action poten- 
tial (SNAP) amplitude was below the 2SD lower limit of normal (LLN; in $3 / 18$ or $16.7 \%$ of patients), based on normative values generated in our Laboratory of Clinical Neurophysiology, while at the same time we found an alteration of Sudoscan profiles in 2 patients (11.1\%) (Figure 1).

After 6 months of treatment, the NCS profiles (especially mean sural SNAP) were altered in $13(72.2 \%)$ patients, and the Sudoscan profiles were modified in $11(61.1 \%)$ subjects (Figure 1).

Employing the McNemar test, the NCS results at baseline and after 6 months were significantly different $(p=0.002)$. Analogously, Sudoscan analysis before and after chemotherapy was statistically different ( $p=0.004)$.

Using the Wilcoxon test for comparison between time points (baseline and after 6 months) of the scales for the study of neuropathy, we observed a significant change after therapy compared to baseline for the DN4 ( $Z=-3.071, p=0.002)$, for the PINRS $(Z=-2.612, p=0.009)$, and for the TNSC $(Z=-2.992, p=0.003)$. These results indicate that bortezomib therapy caused an initial polyneuropathy both clinically and neurophysiologically.

We found a significant correlation between the results obtained with the NCS study or Sudoscan and the scales for the evaluation of neuropathy (Figures 2, 3 - box plot). In particular, using the Spearman test, we found that at baseline NCS correlates with the results of DN4 $\left(r_{s}=0.578\right.$, $p=0.01)$, PINRS $\left(r_{s}=0.482, p=0.04\right)$, and TNSC $\left(r_{s}=0.593, p=0.009\right)$. The correlation was maintained even after 6 months of treatment but only between NCS and DN4 $\left(r_{s}=0.485, p=0.041\right)$.

A significant and positive correlation was also found between the baseline values obtained with

A

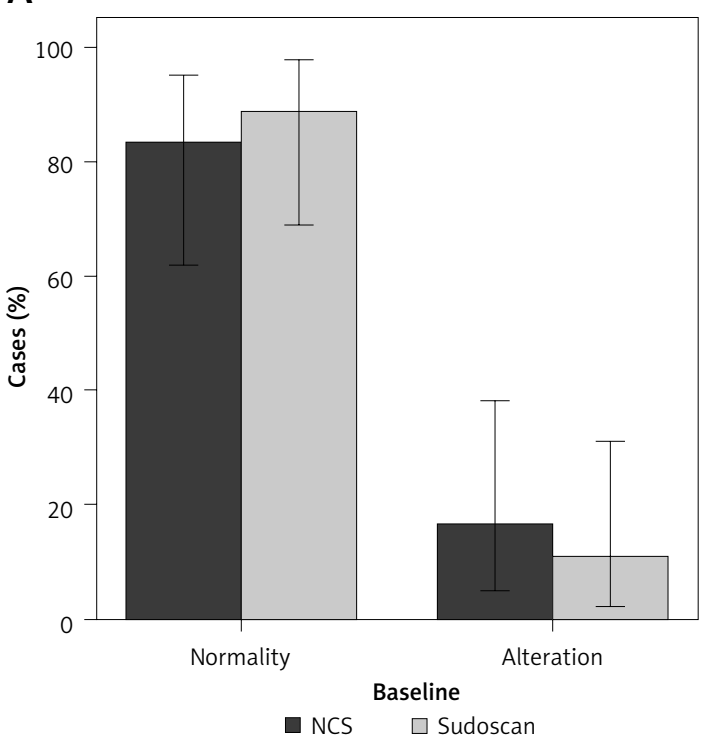

Sudoscan and the scales for neuropathy, specifically with the DN4 $\left(r_{s}=0.469, p=0.049\right)$, with the PINRS $\left(r_{s}=0.500, p=0.03\right)$ and with the TNSc $\left(r_{s}=0.528, p=0.024\right)$. A better correlation was found between Sudoscan and neuropathy scales after treatment with respect to NCS. Indeed, after therapy there was a significant correlation between Sudoscan and DN4 $\left(r_{s}=0.768, p=0.001\right)$, with PINRS $\left(r_{s}=0.824, p=0.0001\right)$, and with TNSc $\left(r_{s}=0.751, p=0.0001\right)$. This different correlation could indicate that Sudoscan is an adequate method to highlight an initial polyneuropathy. No correlation emerged between NCS or Sudoscan and the biological variables considered $(\mathrm{Hb}$, creatinine, LDH, Beta2m, Ig class, light chain, presence of lithic lesions, stage of disease). The only exception was a negative correlation between NCS after 6 months of therapy and calcemia values $\left(r_{s}=-0.574, p=0.013\right)$.

Finally, using the Pearson $\chi^{2}$ test, no statistically significant differences were found between the two techniques used, NCS and Sudoscan at baseline $\left(\chi^{2}=0.232, p=0.630\right)$ and after 6 months of treatment $\left(r_{s}=0.500, p=0.480\right)$.

No difference was detectable for the variables examined between patients subjected to VTD and those subjected to the VMP regimen.

\section{Discussion}

To the best of our knowledge, this study is the first to assess sudomotor dysfunction in patients with MM using a Sudoscan. The primary research objective was to evaluate whether Sudoscan could identify variations of its parameters in $\mathrm{MM}$ patients at diagnosis and after bortezomib treatment. The

\section{B}

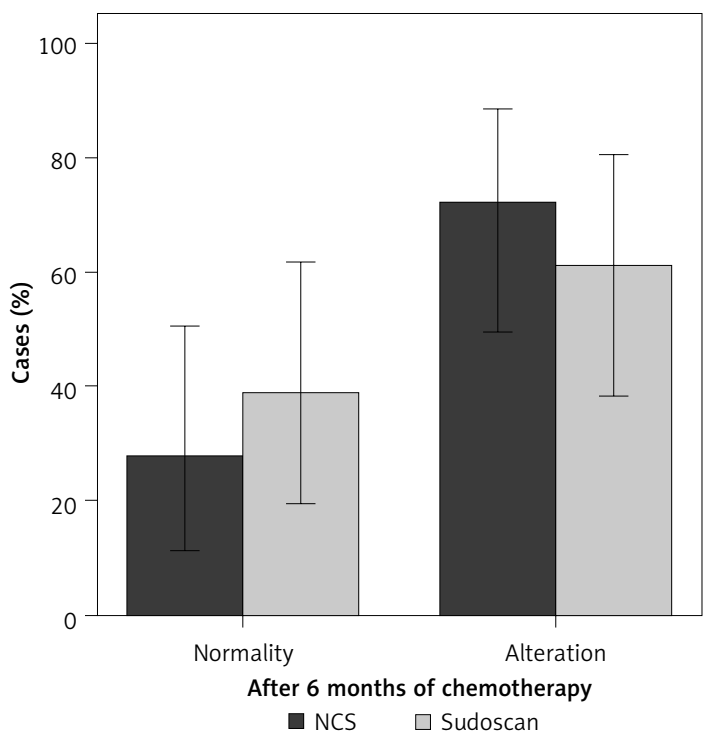

Figure 1. NCS and Sudoscan profiles at baseline and after treatment. "Normality" and "Alterations" indicate, respectively, the absence and the presence of alterations found in NCS and Sudoscan 
A

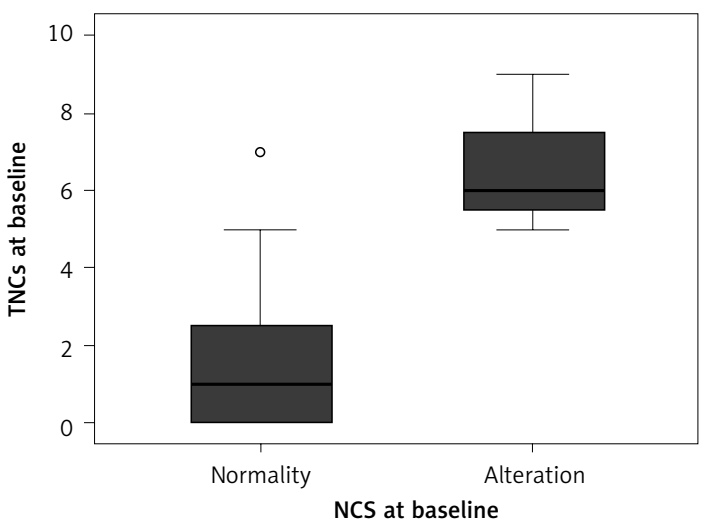

C

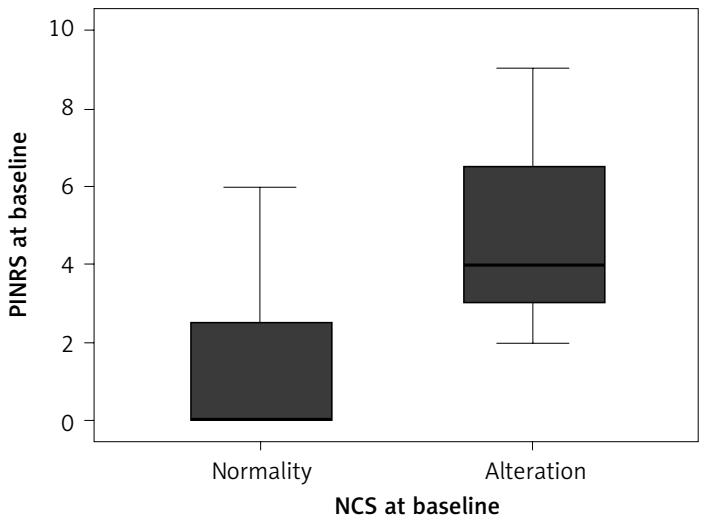

B

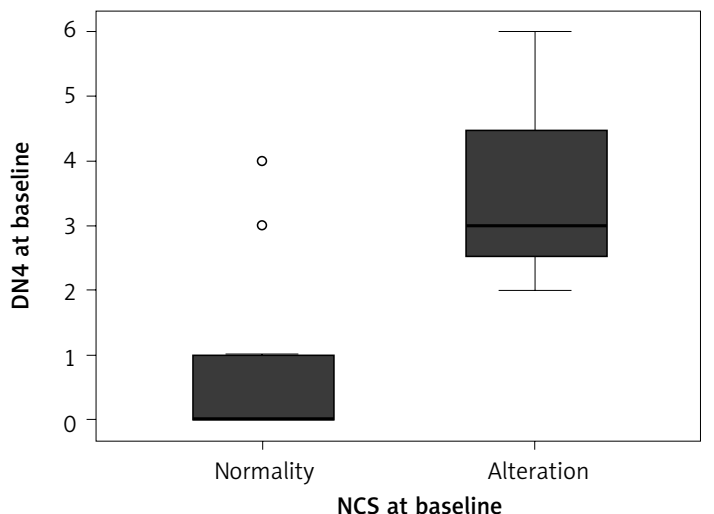

D

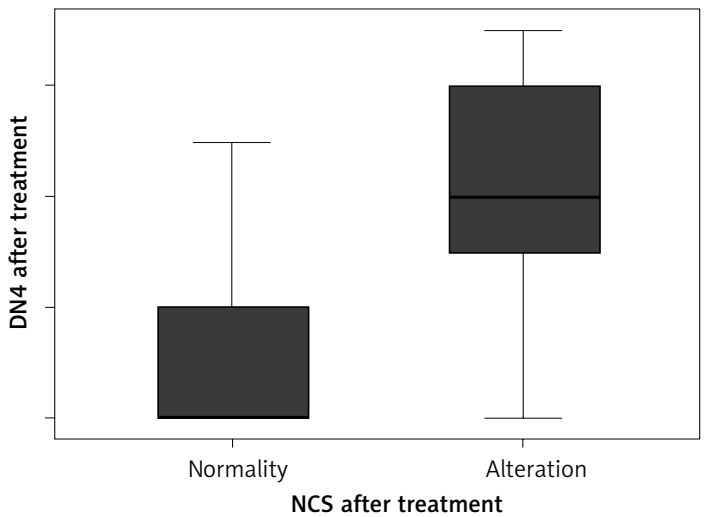

Figure 2. Box plot of DN4 values and PINRS values compared with changing in NCS before and after treatment with bortezomib. "Normality" and "Alterations" indicate, respectively, the absence and the presence of alterations

secondary objective was to evaluate the diagnostic validity of the results and establish the clinical usefulness with respect to conventional NCS.

In our study, we demonstrated for the first time the non-inferiority of the Sudoscan compared to the NCS, in the diagnosis of bortezomib-induced neuropathy in $M M$ patients, evidencing that at the baseline and after therapy there are no statistically significant difference between the two techniques.

Indeed, there seems to be better agreement between Sudoscan and scales for the evaluation of neuropathy with respect to the correlation between scale used and NCS. In fact, after treatment the NCS correlates only with the TNSC, while the Sudoscan maintains a significant correlation with all the scales used. These data could confirm the validity of the technique for the evaluation of bortezomib neuropathy in patients with MM.

The initial identification of neuropathy in clinical practice has been a challenging problem [33]. Conventional assessment techniques, such as the tuning fork and the 10-g monofilament, are subjective and necessitate the patient's collaboration [34, 35]. Generally, a conclusive neuropathy diagnosis is made based on examinations such as NCS, which reveal the alteration of large myelin- ated fibers. However, the findings could be sometimes normal in subjects with early or small fiber prevalent disease [36].

Alterations in peripheral autonomic nervous system conduction are a precocious expression of distal small fiber neuropathy, as sudomotor dysfunction is the initial demonstrable neurophysiologic modifications in distal small fiber neuropathies. For this reason, the American Association of Clinical Endocrinologists proposed sudomotor evaluation as the best tool to evidence early peripheral autonomic neuropathy in diabetic patients.

Currently, there are several techniques of evaluating sudomotor function [37], but unfortunately, none are appropriate for application in daily clinical practice due to the needs of highly prepared operators, protracted investigating time, expensive apparatus, and difficult subject training [38]. The possible employment of Sudoscan seems to resolve all these issues, as it can be executed in a few minutes, does not need any subject collaboration or specific preparation of the operators, and it is absolutely noninvasive.

BIPN is characteristically sensory, and the main symptoms are itchiness, hyperesthesia or hypoesthesia, pain or burning sensations [11, 39]. However, motor polyneuropathy from bortezomib is 
A

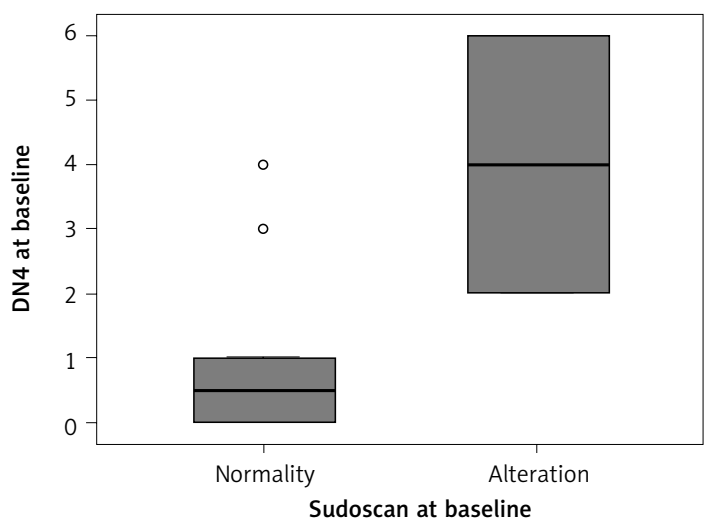

C

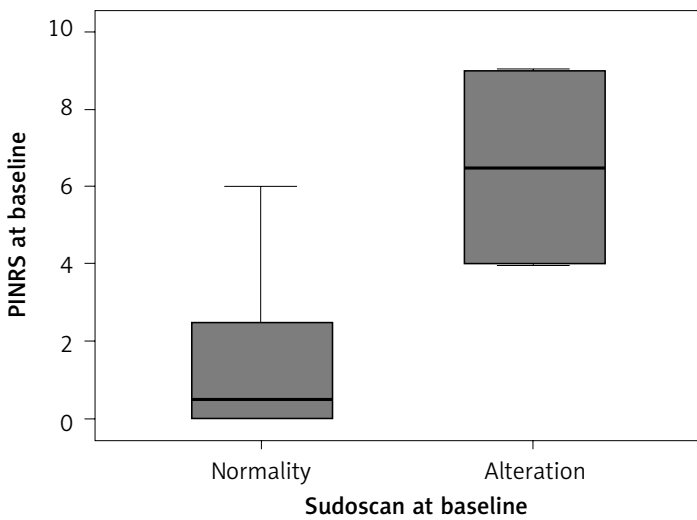

$\mathrm{E}$

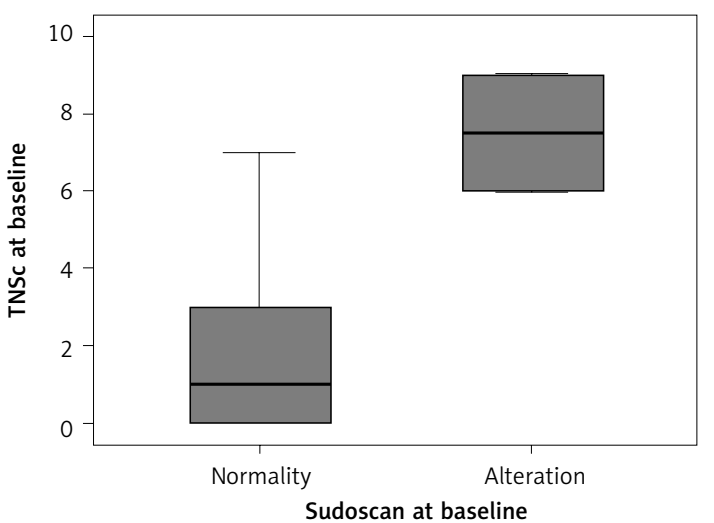

B

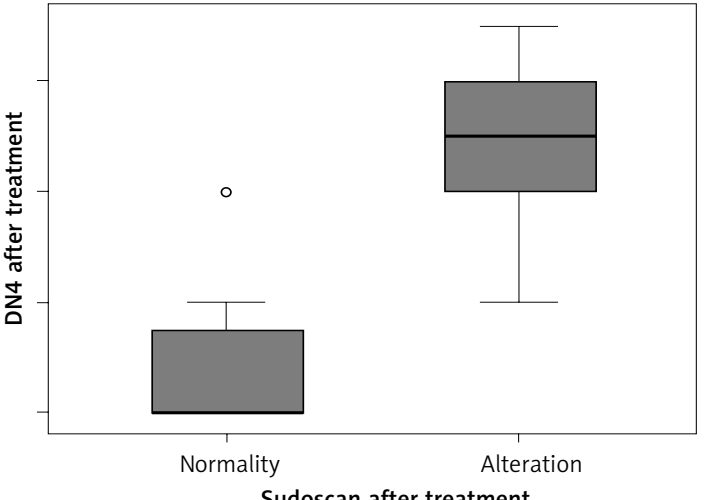

Sudoscan after treatment

D

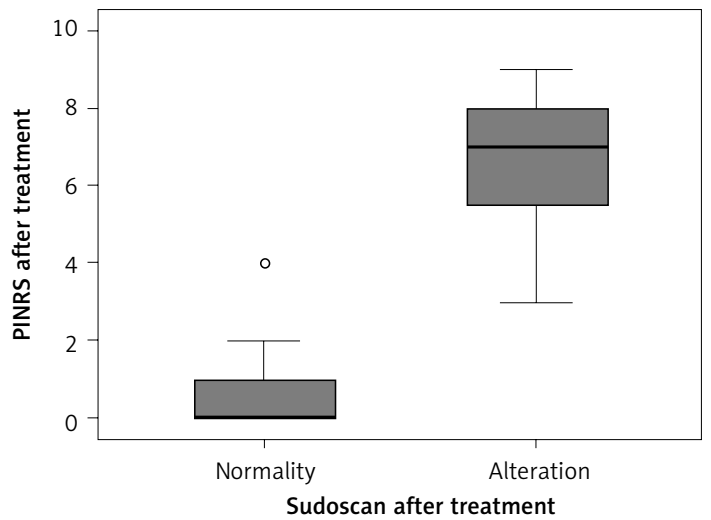

$\mathrm{F}$

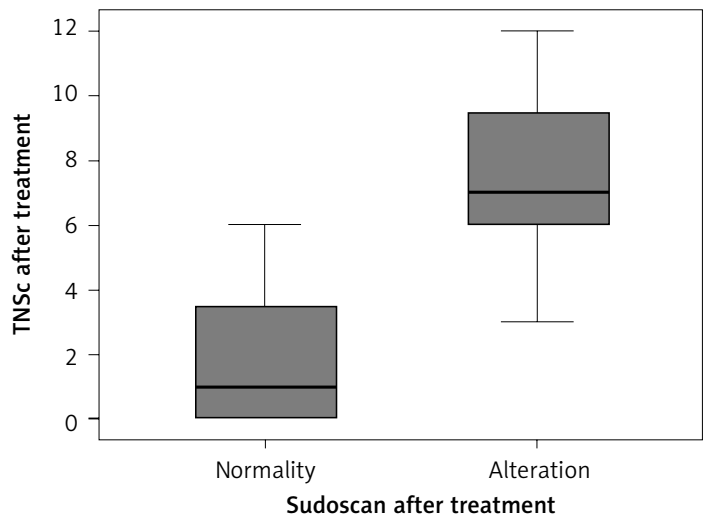

Figure 3. Box plot of DN4, PINRS and TNSc values compared with changing in Sudoscan before and after treatment with bortezomib. "Normality" and "Alterations" indicate, respectively, the absence and the presence of alterations

infrequent and generally appears after sensory polyneuropathy.

The pathogenetic mechanism of BIPN has not yet been distinctly described. Several results have indicated the existence of genetic factors [40]. Certain pathways might have a central action in BIPN, such as NFאB, DNA repair, and mitogen-activated protein kinase-mediated signaling [41-43]. Bortezomib has also been demonstrated to act on mitochondria, leading to a decrease in mitochondrial membrane potential and augmented reactive oxygen production (ROS) in vitro [44]. These observations and the well-known alteration of oxidative stress in MM patients [45] support the possibility that in vivo ROS scavenging may be a successful target in blocking BIPN.

Finally, there are signs of calcium dysregulation in bortezomib-caused cell death, with bortezomib provoking temporary intracellular ER calcium release, causing a mitochondrial-calcium influx and caspase activation [46]. This temporary modification may modify the membrane potential of neurons and provoke spontaneous action potentials, generating pain signaling after bortezomib treatment [47]. 
In this regard, our detection of a negative relationship between serum calcium levels and NCS after treatment with bortezomib $(-0.574, p=0.05)$ seems to be worthy of in-depth analysis.

Recent reports have demonstrated the diagnostic validity of ESC evaluation, with a sensitivity of $73 \%$ to $78 \%$, and specificity of $62 \%$ to $100 \%$ to evidence PPNP in diabetic patients as well as idiopathic polyneuropathy (PN) and oxaliplatin or paclitaxel chemotherapy-induced PN [48-50], and previous studies assessing the Sudoscan risk score registered $65-92 \%$ sensitivity and $49-80 \%$ specificity to evidence cardiac autonomic neuropathy [51].

Our results suggest that sudomotor function, assessed by Sudoscan, can be considered for the diagnosis of bortezomib-induced neuropathy. Sudoscan analysis is painless, can be conducted in a few minutes, and neither special patient preparation nor specially trained operators are needed. It is objective, reproducible, and surely easier than the traditional NCS.

However, our study has some limitations. In fact, the number of subjects studied is too small to be able to draw definitive and generalizable conclusions, and a longer follow-up of the patients would be appropriate. Nevertheless, in view of the pathophysiology of BIPN, which suggests the predominant participation of small fibers in the onset of the disease, it is probable that subsequent studies conducted on a larger population will demonstrate even better results regarding the sensitivity and specificity of Sudoscan compared to the NCS.

So, Sudoscan may become a novel technique for diagnosis of neuropathy in daily activity, and a useful help to manage the therapeutic interventions in MM.

\section{Conflict of interest}

The authors declare no conflict of interest.

\section{References}

1. Allegra A, Alonci A, Gerace D, et al. New orally active proteasome inhibitors in multiple myeloma. Leuk Res 2014; 38: 1-9.

2. Allegra A, Penna G, Alonci A, et al. Monoclonal antibodies: potential new therapeutic treatment against multiple myeloma. Eur J Haematol 2013; 90: 441-68.

3. Allegra A, Sant'antonio E, Penna G, et al. Novel therapeutic strategies in multiple myeloma: role of the heat shock protein inhibitors. Eur J Haematol 2011; 86: $93-$ 110.

4. Yang $M$, Zhang L, Wang X, Zhou Y, Wu S. Down-regulation of miR-203a by IncRNA PVT1 in multiple myeloma promotes cell proliferation. Arch Med Sci 2018; 14: 1333-9.

5. Kumar SK, Dispenzieri A, Lacy MQ, et al. Continued improvement in survival in multiple myeloma: changes in early mortality and outcomes in older patients. Leukemia 2014; 28: 1122-8.

6. Ettari R, Zappalà M, Grasso S, Musolino C, Innao V, Allegra A. Immunoproteasome-selective and non-selective inhibitors: a promising approach for the treatment of multiple myeloma. Pharmacol Ther 2018; 182: 176-92.

7. Staff NP, Podratz JL, Grassner L, et al. Bortezomib alters microtubule polymerization and axonal transport in rat dorsal root ganglion neurons. Neurotoxicology 2013; 39: 124-31.

8. Voorhees PM, Orlowski RZ. The proteasome and proteasome inhibitors in cancer therapy. Annu Rev Pharmacol Toxicol 2006; 46: 189-213.

9. Voorhees PM, Laubach J, Anderson KC, Richardson PG. Peripheral neuropathy in multiple myeloma patients receiving lenalidomide, bortezomib, and dexamethasone (RVD) therapy. Blood 2013; 121: 858.

10. Seretny M, Currie GL, Sena, ES, et al. Incidence, prevalence, and predictors of chemotherapy-induced peripheral neuropathy: a systematic review and meta-analysis. Pain 2014; 155: 2461-70.

11. Delforge M, Bladé J, Dimopoulos MA, et al. Treatment-related peripheral neuropathy in multiple myeloma: the challenge continues. Lancet Oncol 2010; 11: 1086-95.

12. Richardson PG, Sonneveld P, Schuster MW, et al. Reversibility of symptomatic peripheral neuropathy with bortezomib in the phase III APEX trial in relapsed multiple myeloma: impact of a dose-modification guideline. $\mathrm{Br}$ J Haematol 2009;144: 895-903.

13. Cavaletti G, Jakubowiak AJ. Peripheral neuropathy during bortezomib treatment of multiple myeloma: a review of recent studies. Leuk Lymphoma 2010; 51: 1178-87.

14. Boyette-Davis JA, Cata JP, Zhang H, et al. Follow-up psychophysical studies in bortezomib-related chemoneuropathy patients. J Pain 2011; 12: 1017-24.

15. Cavaletti G, Nobile-Orazio E. Bortezomib-induced peripheral neurotoxicity: still far from a painless gain. Haematologica 2007; 92: 1308-10.

16. Dyck PJ, O’Brien PC, Litchy WJ, Harper CM, Klein CJ, Dyck PJ. Monotonicity of nerve tests in diabetes: sub-clinical nerve dysfunction precedes diagnosis of polyneuropathy. Diabetes Care 2005; 28: 2192-200.

17. Weber F, Albert U. Electrodiagnostic examination of lumbosacral radiculopathies. Electromyogr Clin Neurophysiol 2000; 40: 231-6.

18. Spallone V, Ziegler D, Freeman R, et al. Cardiovascular autonomic neuropathy in diabetes: clinical impact, assessment, diagnosis, and management. Diabetes Metab Res Rev 2011; 27: 639-53.

19. McArthur JC, Stocks EA, Hauer P, Cornblath DR, Griffin JW. Epidermal nerve fiber density: normative reference range and diagnostic efficiency. Arch Neurol 1988; 55: 1513-20.

20. Fealey RD, Low PA, Thomas JE. Thermoregulatory sweating abnormalities in diabetes mellitus. Mayo Clin Proc 1989; 64: 617-28.

21. Casellini CM, Parson HK, Richardson MS, Nevoret ML, Vinik Al. Sudoscan, a noninvasive tool for detecting diabetic small fiber neuropathy and autonomic dysfunction. Diabetes Technol Ther 2013; 15: 948-53.

22. Mayaudon H, Miloche PO, Bauduceau B. A new simple method for assessing sudomotor function: relevance in type 2 diabetes. Diabetes Metab 2010; 36: 450-4.

23. Gin H, Baudoin R, Raffaitin CH, Rigalleau V, Gonzalez C. Non-invasive and quantitative assessment of sudomotor function for peripheral diabetic neuropathy evaluation. Diabetes Metab 2011; 37: 527-32. 
24. Luigetti M, Primiano G, Cuccagna C, et al. Small fibre neuropathy in mitochondrial diseases explored with sudoscan. Clin Neurophysiol 2018; 129: 1618-23.

25. Lefaucheur JP, Zouari HG, Gorram F, Nordine T, Damy T, Planté-Bordeneuve V. The value of electrochemical skin conductance measurement using SudoscanVR in the assessment of patients with familial amyloid polyneuropathy. Clin Neurophysiol 2018; 129: 1565-9.

26. Van Den Kerkhof EG, Stitt L, Clark AJ, et al. Sensitivity of the DN4 in screening for neuropathic pain syndromes. Clin J Pain 2018; 34: 30-6.

27. Zilliox LA, Ruby SK, Singh S, Zhan M, Russell JW. Clinical neuropathy scales in neuropathy associated with impaired glucose tolerance. I Diabetes Complications 2015; 29: 372-7.

28. Williamson A, Hoggart B. Pain: a review of three commonly used pain rating scales. J Clin Nurs 2005; 14 798-804.

29. Vinik Al, Nevoret ML, Casellini C. The new age of sudomotor function testing: a sensitive and specific biomarker for diagnosis, estimation of severity, monitoring progression, and regression in response to intervention. Front Endocrinol 2015; 6: 94.

30. Chizmadzhev YA, Indenbom AV, Kuzmin PI, Galichenko SV, Weaver JC, Potts RO. Electrical properties of skin at moderate voltages: contribution of appendageal macropores. Biophys J 1998; 74: 843-56.

31. Brunswick P, Mayaudon H, Albin V, Lair V, Ringuede A, Cassir M. Use of Ni electrodes chronoamperometry for improved diagnostics of diabetes and cardiac diseases. Conf Proc IEEE Eng Med Biol Soc 2007; 2007: 4544-7.

32. Wisotsky E, Tseng V, Pohlman D. Pocket EMG. 2014; 176. Gasch: USA.

33. Quattrini C, Jeziorska M, Malik RA. Small fiber neuropathy in diabetes: clinical consequence and assessment. Int J Low Extrem Wounds 2004; 3: 16-21.

34. Perkins BA, Olaleye D, Zinman B, Bril V. Simple screening tests for peripheral neuropathy in the diabetes clinic. Diabetes Care 2001; 24: 250-6.

35. Papanas N, Ziegler D. New vistas in the diagnosis of diabetic polyneuropathy. Endocrine 2014; 47: 690-8.

36. Gronseth JDEGS. Distal symmetric polyneuropathy a definition for clinical research report of the American Academy of Neurology, the American Association of Electrodiagnostic Medicine, and the American Academy of Physical Medicine and Rehabilitation. Neurology 2005; 64: 199-207.

37. Kempler P, Amarenco G, Freeman R, et al.; the Toronto Consensus Panel on Diabetic Neuropathy. Gastrointestinal autonomic neuropathy, erectile, bladder-and sudomotor dysfunction in patients with diabetes mellitus: clinical impact, assessment, diagnosis, and management. Diabetes Metab Res Rev 2011; 27: 665-77.

38. Dyck PJ, Overland CJ, Low PA, et al. Signs and symptoms versus nerve conduction studies to diagnose diabetic sensorimotor polyneuropathy: Cl vs. NPhys trial. Muscle Nerve 2010; 42: 157-64.

39. Badros A, Goloubeva O, Dalal JS, et al. Neurotoxicity of bortezomib therapy in multiple myeloma: a single-center experience and review of the literature. Cancer 2007; 110: 1042-9.

40. Chubb D, Weinhold N, Broderick P, et al. Common variation at 3q26.2, 6p21.33, 17p11.2 and 22q13.1 influences multiple myeloma risk. Nat Genet 2013; 45: 1221-5.

41. Mitchell JS, Li N, Weinhold N, et al. Genome-wide association study identifies multiple susceptibility loci for multiple myeloma. Nat Commun 2016; 7: 12050.
42. Campo C, Da Silva Filho MI, Weinhold N, et al. Genetic susceptibility to bortezomib-induced peripheral neuropathy: replication of the reported candidate susceptibility loci. Neurochem Res 2017; 42: 925-31.

43. Du J, Huo J, Shi J, et al. Polymorphisms of nuclear factor-kappa B family genes are associated with development of multiple myeloma and treatment outcome in patients receiving bortezomib-based regimens. Haematologica 2011; 96: 729-37.

44. Maharjan S, Oku M, Tsuda M, Hoseki J, Sakai Y. Mitochondrial impairment triggers cytosolic oxidative stress and cell death following proteasome inhibition. Sci Rep 2014; 4: 5896.

45. Gangemi S, Allegra A, Alonci A, et al. Increase of novel biomarkers for oxidative stress in patients with plasma cell disorders and in multiple myeloma patients with bone lesions. Inflamm Res 2012; 61: 1063-7.

46. Landowski TH, Megli CJ, Nullmeyer KD, Lynch RM, Dorr RT. Mitochondrial-mediated disregulation of $\mathrm{Ca} 2+$ is a critical determinant of Velcade (PS-341/Bortezomib) cytotoxicity in myeloma cell lines. Cancer Res 2005; 65: 3828-36.

47. Robinson CR, Zhang H, Dougherty PM. Altered discharges of spinal neurons parallel the behavioral phenotype shown by rats with bortezomib related chemotherapy induced peripheral neuropathy. Brain Res 2014; 1574: 6-13.

48. Selvarajah D, Cash T, Davies J, et al. Sudoscan: a simple, rapid, and objective method with potential for screening for diabetic peripheral neuropathy. PLoS One 2015; 10: e0138224.

49. Smith AG, Lessard M, Reyna S, Doudova M, Singleton JR. The diagnostic utility of Sudoscan for distal symmetric peripheral neuropathy. J Diabetes Complications 2014; 28: 511-6.

50. Saad M, Psimaras D, Tafani C, et al. Quick, non-invasive and quantitative assessment of small fiber neuropathy in patients receiving chemotherapy. J Neurooncol 2016; 127: 373-80.

51. Yajnik CS, Kantikar V, Pande A, et al. Screening of cardiovascular autonomic neuropathy in patients with diabetes using non-invasive quick and simple assessment of sudomotor function. Diabetes Metab 2013; 39: 126-31. 